\title{
Análisis de factores de capacidad institucional en municipios pequeños de los departamentos de Boyacá y Cundinamarca*
}

Analysis of institutional capacity factors in small municipalities in the departments of Boyacá and Cundinamarca

Analyse des facteurs de capacité institutionnelle dans de petites municipalités des départements de Boyacá et de Cundinamarca

\section{Resumen}

La introducción de una cultura de la gestión en el funcionamiento de los organismos y entidades del Estado ha conducido a una preocupación constante por el fortalecimiento de la capacidad institucional, que desde las diferentes perspectivas teóricas puede abordarse a partir de varios aspectos como los procesos, los resultados y/o el contexto. En Colombia se ha enfocado en los resultados y esta preocupación por los resultados ha dado origen a la "Metodología para la medición y análisis del desempeño municipal" que busca verificar el cumplimiento de estas entidades territoriales en términos de eficiencia, eficacia y cumplimiento de requisitos legales. Esta medición, aunque refleja buenos resultados en el desempeño de un número importante de municipios, deja de lado el análisis de factores relacionados con la dotación de recursos humanos, la relación entre recursos y funciones, así como las verdaderas posibilidades de acumulación de capacidad, sobre todo para los municipios pequeños. El análisis realizado con una muestra de 10 municipios, de los cuales cinco (5) son del departamento de Cundinamarca y cinco (5) del departamento de Boyacá, permitió evidenciar debilidades importantes relacionadas en primer lugar con la presencia en un alto porcentaje de recurso humano cuya formación no está relacionada con los asuntos que son propios de la administración pública, lo que obliga al aprendizaje en la práctica. Además, no hay un proyecto claro en materia de capacitación y acompañamiento que le permita al personal existente la formación y/o actualización permanente frente a las diferentes temáticas de la gestión pública. Esta situación resulta fundamental en la medida que las funciones de los municipios se relacionan con una multiplicidad de sectores que demuestran niveles importantes de complejidad y transformación. Asimismo, los servidores públicos mejor preparados son de libre nombramiento y remoción, lo cual condena a estas entidades a niveles cíclicos de acumulación y disminución de capacidad institucional.

Artículo de investigación científica y tecnológica Según Clasificación Colciencias

Fecha de recepción: 27/02/2012

Fecha de corrección: 25/04/2012

Fecha de aprobación: 20/05/2012

Palabras clave: Municipios, gestión local, administración pública, descentralización, capacidad institucional.

* Este artículo es producto del proyecto de investigación "Capacidad Institucional de los municipios pequeños en Colombia", financiado en su totalidad por la Institución Universitaria Politécnico Grancolombiano. 
Analysis of institutional capacity factors in small municipalities in the departments of Boyacá and Cundinamarca

\author{
Análisis de factores de capacidad \\ institucional en municipios pequeños de los \\ departamentos de Boyacá y Cundinamarca \\ Analyse des facteurs de capacité \\ institutionnelle dans de petites \\ municipalités des départements de Boyacá \\ et de Cundinamarca
}

\section{Abstract}

The introduction of a management culture in the operation of state agencies and institutions has led to a constant concern for strengthening institutional capacity, which, although from different theoretical perspectives can be approached from several aspects such as processes, results and / or context, in our environment has focused on results. In Colombia this concern for the results has given rise to the "Methodology for Measurement and Analysis of Municipal Performance" Which seeks to verify compliance with these local entities in terms of efficiency, effectiveness and compliance with legal requirements. This measurement shows good performance for many municipalities, but does not take into account the analysis of factors related to the supply of human resources, the relationship between resources and functions and the real possibilities of capacity building, particularly for small municipalities.

The analysis of a sample of 10 municipalities, of which five are from the department of Cundinamarca and five from the department of Boyacá, shows major weaknesses, which are related, first, with a high percentage of staff whose training is not related to public administration, forcing them to learn from practical experience. Additionally, there is not a clear plan for training and support that will enable existing staff training and / or permanent updating about public management issues. This situation is critical to the extent that the municipal functions relate multiple sectors which demonstrate significant levels of complexity and evolution. Similarly, better prepared public servants are free appointment and removal, which condemns these entities to cyclical levels of accumulation and decreased institutional capacity.

Keywords: municipalities, municipal governance, public administration, decentralization, institutional capacity.

\section{Analyse des facteurs de capacité institutionnelle dans de petites municipalités des départements de Boyacá et de Cundinamarca}

\author{
Análisis de factores de capacidad \\ institucional en municipios pequeños de los \\ departamentos de Boyacá y Cundinamarca
}

Analysis of institutional capacity factors in small municipalities in the departments of Boyacá and Cundinamarca

\section{Résumée}

L'introduction d'une culture de la gestion dans le fonctionnement des organismes et des entités de l'État, a conduit à une préoccupation constante pour le renforcement de la capacité institutionnelle, qui depuis les différentes perspectives théoriques peut être abordée à partir de plusieurs aspects tels que les processus, les résultats et/ou le contexte. En Colombie, l'approche porte sur les résultats et cette préoccupation pour les résultats a donné l'origine à la "Méthodologie pour la mesure et l'analyse du développement municipal”. Celle-ci cherche à vérifier l'accomplissement de ces entités territoriales en termes d'efficacité et de réalisation des exigences légales. Quoique cette mesure reflète de bons résultats dans le développement d’un nombre important de municipalités, elle ne tient pas compte de l'analyse des facteurs liés à la dotation de ressources humaines, la relation entre ressources et fonctions, ainsi que les véritables possibilités d'accumulation de capacité, surtout pour les petites municipalités. L'analyse réalisé dans une dizaine de municipalités, dont cinq font partie du département de Cundinamarca et cinq de celui de Boyacá, a permis de mettre en évidence des faiblesses importantes touchant en premier lieu la présence en fort pourcentage de ressources humaines dont la formation n'est pas liée aux sujets propres de l'administration publique, ce qui oblige l'apprentissage sur la pratique. En plus, il nexiste pas de projet clair ni en formation ni en accompagnement qui permette au personnel existant la formation et/ou la mise à jour en permanence face aux différentes thématiques de la gestion publique. Cette situation résulte fondamentale, dans la mesure où les fonctions des municipalités sont liées à une multiplicité de secteurs qui démontrent des niveaux importants de complexité et de transformation. De même, les serviteurs publics les mieux préparés sont de libre nomination et remuement, ce qui condamne ces entités à des niveaux cycliques d'accumulation et de diminution de capacité institutionnelle.

Mots clef: municipalités, gestion locale, administration publique, décentralisation, capacité institutionnelle. 


\section{Análisis de factores de capacidad institucional en municipios pequeños de los departamentos de Boyacá y Cundinamarca}

\section{Introducción}

Desde la reforma descentralista en Colombia se ha debatido ampliamente sobre las evidentes debilidades de los municipios pequeños. Estas entidades, que suman un porcentaje bastante alto, han sido el blanco de constantes críticas enfocadas principalmente en el plano fiscal. Aunque desde la filosofía del proceso de transformación del Estado la descentralización puso en primer plano al municipio por ser el escenario natural de interlocución directa entre el Estado y la sociedad, la realidad ha demostrado que el diseño normativo no ha sido consecuente con las limitaciones y posibilidades que ofrecen los municipios en materia de capacidad institucional.

Además, las lógicas de medición del desempeño que imperan actualmente en el sector público están guiadas por principios provenientes de la nueva gestión pública, que privilegian sobre todo los resultados, dejando de lado otro tipo de factores que podrían ser determinantes a la hora de reflexionar sobre la conveniencia o no de los municipios pequeños. En este sentido, este artículo ofrece un análisis de la realidad administrativa de 10 municipios considerados pequeños por ubicarse en las categorías 4, 5 y 6, de los departamentos de Cundinamarca (Arbeláez, Tenjo, Sesquilé, Suesca y Lenguazaque) y Boyacá (Belén, Cómbita, Nobsa, Samacá y Tibasosa), a partir de la identificación de aspectos importantes que permiten describir las condiciones de capacidad instalada con que cuentan estas entidades para el desempeño de sus funciones. Para ello, se aplicaron alrededor de 30 entrevistas a los servidores públicos del gabinete de gobierno, alcaldes y secretarios del despacho, y 50 encuestas a otros funcionarios de la administración central de los municipios, para medir el perfil y las percepciones frente a sus cargos.

Tomando en cuenta esta información, así como otro tipo de fuentes académicas y normativas, este artículo se estructuró comenzando con una revisión de las aproximaciones e interpretaciones alrededor del concepto de capacidad institucional, con el fin de identificar el nivel en el que se encuentra ubicada la medición del desempeño en Colombia. El documento ofrece una descripción de las particularidades que encierra la medición de desempeño en el país y la clasificación que recibieron los municipios utilizados para el análisis, a partir de cada uno de los factores considerados en la metodología. Tomando en cuenta los anteriores aspectos, se presenta una descripción de las debilidades estructurales identificadas para los municipios pequeños, en relación con la forma como la dotación de recursos y el agregado de funciones que les corresponden impactan en la estructura administrativa. Por último, se introduce una reflexión alrededor de los factores externos a la administración pero que constituyen el fundamento principal a la hora de pensar en su fortalecimiento y que deben conducir a la concepción del municipio más allá dela lógica de medición imperante.

\section{Aproximaciones al concepto de capacidad institucional}

El interés por la capacidad institucional del sector público forma parte de las denominadas "reformas de segunda generación" del Estado. Luego del primer paquete de reformas que buscaban la estabilización fiscal de los estados latinoamericanos, y que condujo a la preminencia del mercado, el segundo momento de la transformación se enfocó en el ámbito de la administración pública. El propósito del nuevo conjunto de medidas de reforma buscaba cambiar la visión de "menos Estado" a "mejor Estado", logrando con ello poner de nuevo al Estado en un primer plano mediante el fortalecimiento y mejoramiento de la capacidad de gestión de sus organismos y entidades.

De acuerdo con Ospina (2002) un primer momento de este período de ajustes, que se puede ubicar en la segunda mitad de década de1980, se caracterizó por orientarse hacia el mejoramiento de las habilidades del Estado en el cumplimiento de sus funciones mediante el "fortalecimiento institucional" a través de la reingeniería organizacional. El propósito era el mejoramiento de la estructura interna, los sistemas y las estrategias de la organización. La década siguiente dio lugar al concepto de "buen gobierno" como filosofía definitoria de la actuación del Estado y cuya característica fundamental era la construcción de 
capacidad institucional como base del proceso (Ospina, 2002). El concepto proponía la intervención de diferentes actores, públicos, privados y sociales en los procesos de identificación y resolución de los problemas públicos con miras al logro de objetivos de desarrollo (Banco Mundial, 1996).

La primera década del siglo XXI experimentó una variación en el concepto de capacidad institucional, ahora en relación con el de gobernanza, que ha conducido a la concepción de la capacidad de gestión más allá del aparato administrativo estatal para integrar grupos sociales, el entorno y la ciudadanía en general, con el fin de articular la capacidad administrativa con la capacidad política. Esta nueva capacidad incluye la habilidad de las organizaciones para absorber responsabilidades, operar con más eficiencia y fortalecer la rendición de cuentas (Rosas, 2008; Ospina, 2002). En términos generales, la gobernanza se puede entender como un balance de poderes y de acciones a diferentes niveles de autoridad, "que se produce dentro de sistemas políticos, leyes, regulaciones, instituciones, mecanismos financieros y tiende hacia un desarrollo de la sociedad civil” (Domínguez y Jiménez, 2010, p. 2).

Todas estas aproximaciones al concepto de capacidad institucional coinciden en la orientación hacia la adaptación de las organizaciones públicas para responder a los problemas públicos, la implementación de mecanismos para la formulación, aplicación, coordinación, monitoreo, y evaluación de las políticas públicas así como la implementación de mecanismos de rendición de cuentas en el marco de un nuevo sistema de gestión pública (Rosas, 2008).

Asimismo, estos diferentes momentos históricos y aproximaciones al concepto, han conducido a la generación de una escala agregada de factores que han buscado describir el concepto de la capacidad institucional partiendo de una visión simple hasta llegar a definiciones más complejas y sistémicas. En esos términos, la mayoría de las definiciones, en particular aquellas que aparecieron en la primera etapa de estas reformas, hacen referencia a la capacidad institucional como capacidad del aparato administrativo público desde una perspectiva técnico-burocrática, sin considerar los factores del entorno.

En este tipo de interpretaciones, cuyo énfasis se encuentra puesto en los medios y en los procesos administrativos, se pueden mencionar autores como Migdal (1998) quien habla de la capacidad institucional como aquella que tiene los líderes estatales de utilizar los órganos del Estado con el fin de que se cristalicen sus decisiones en el seno de la sociedad. En esta misma línea encontramos a Grindle (1997) y Evans (1996) cuyo énfasis está puesto en las características y el desarrollo de los recursos humanos como factores determinantes para el desarrollo y eficacia del aparato organizacional estatal.

Por su parte, Weiss (1998) define la capacidad institucional como la capacidad transformativa del Estado entendida como la habilidad para adaptarse a los choques y a las presiones externas, y Willems (2004) que la define como la habilidad de las instancias gubernamentales de mejorar las funciones, de resolver problemas y especificar y lograr objetivos y como la habilidad de movilizar o adaptar las instituciones para dar respuesta a un problema público. Aunque estas últimas definiciones toman en cuenta el contexto y la necesidad de la adaptación de los aparatos gubernamentales al mismo, su énfasis se encuentra puesto en el funcionamiento administrativo interno de las organizaciones públicas.

Complementarios con estos planteamientos es posible identificar otras posiciones que aunque no dejan de lado los medios y los procesos internos, le confieren mayor importancia a los resultados, entendidos desde la óptica administrativista. En este sentido, Hilderbrand y Grindle (1997, p. 34) plantean que "capacidad es la habilidad para desempeñar tareas de una manera efectiva, eficiente y sostenible", similar a Chávez y Rayas (2006, p. 2) quienes consideran que la capacidad institucional se refiere:

al desarrollo de la estructura de toda institución que le permita asumir sus responsabilidades de manera ordenada y coordinada, en el corto, mediano y largo plazo. Es decir, la eficacia y eficiencia de los procesos institucionales, considerando la realización de las tareas y su grado de cumplimiento.

Estos planteamientos, aunque tomen en cuenta el entorno y los procesos internos, ponen especial énfasis en los resultados de la organización, los cuales dentro de la perspectiva actual, se encuentran guiados por los principios y valores que identifican a la nueva gestión pública, como son la eficiencia, la eficacia, la transparencia y la orientación al usuario. En estos enfoques el con- 
texto y los elementos y actores que lo definen, se presentan como factores a tener en cuenta para configurar la organización en términos de funcionalidad administrativa para el cumplimiento de metas e indicadores de desempeño.

Finalmente, están los planteamientos que se refieren a la capacidad institucional en términos de resultados sociales y políticos como es el caso de Reppeto (2007), quien se refiere a la capacidad institucional como "la aptitud de las instancias gubernamentales de plasmar en las políticas públicas los máximos niveles posibles de valor social". Para este autor es más importante el para qué que el cómo, por lo que considera que aunque la "capacidad administrativa" es condición necesaria, no es suficiente para lograr niveles relevantes de capacidad estatal. Para ello plantea lo que denomina "capacidad política" y que interpreta como:

la capacidad de los gobernantes para problematizar las demandas de los grupos mayoritarios de la población, tomando decisiones que representen y expresen los intereses e ideologías de los mismos más allá de la dotación de recursos que puedan movilizar en la esfera pública (Reppeto, 2007, p. 54).

En esta misma perspectiva se ubican las posiciones sobre gobernabilidad cuyo énfasis se encuentra puesto en "la capacidad de dirección y de coordinación de los gobiernos tan turbulentos -governance- diferenciándose entre aquello que atañe al colectivo sobre el cual se ejerce la acción de gobierno y aquello que atañe a la acción misma de gobernar" (Brito, 2002, p. 10). La gobernabilidad entraña la idea que la acción de las autoridades sobre los ciudadanos corresponde con los criterios y principios, producto de las vivencias y el aprendizaje colectivo, y se expresa mediante normas y políticas que son válidas para el conjunto social (Arbós y Giner, 1993).

La importancia de la capacidad institucional desde las diferentes perspectivas es, a su vez, contemporánea con el énfasis sobre el fortalecimiento de los niveles locales de gobierno, lo cual ha conducido a considerar que la descentralización es un mecanismo tanto de democratización como de fortalecimiento de la gestión del Estado. Este vínculo surgió de la denominada "crisis del centralismo" que puso en evidencia los defectos de un modelo de administración pública inadecuado y anacrónico frente a las caracterís- ticas del nuevo contexto globalizado. El excesivo centralismo había enaltecido al nivel nacional y rezagado el ámbito de lo local de los escenarios administrativos y políticos relativos al quehacer del Estado, convirtiendo a sus organismos y autoridades públicas en decisores y ejecutores miopes e ineficientes frente a la realidad que buscaban intervenir. La reforma implicó la transformación radical de la configuración de las estructuras estatales, mediante una redistribución del poder y una reasignación de funciones y responsabilidades entre la nación y las entidades territoriales del nivel local.

El proceso mismo se consideró un paso necesario y urgente para garantizar una adecuada readaptación del Estado con el fin de garantizar su permanencia como ámbito indispensable de representación social. Para ello era necesario fortalecerlo desde la base y reducir el protagonismo centenario de los otros niveles del Estado. Esta urgencia, que en realidad respondió más a propósitos de tipo político que administrativo, impidió vislumbrar, en un primer momento los requerimientos y los niveles existentes en materia de capacidad administrativa. Pero, además de la falta de previsión en términos de dotación institucional frente a los niveles iniciales requeridos antes de la descentralización y la ausencia de un proyecto claro de fortalecimiento de capacidades, el proceso ha enfatizado en la descentralización del gasto, soportado en las transferencias, antes que en la descentralización del ingreso mediante la ampliación de potestades recaudatorias (Cabrero, 2006).

Todos estos elementos han conducido en la práctica a un déficit permanente de capacidad para las administraciones locales de varios países en Latinoamérica, incluido Colombia, debido a las presiones administrativas y fiscales derivadas de la nueva carga de responsabilidades. Sin embargo, frente a las expectativas y exigencias surgidas del nuevo paradigma orientado a la gestión, que todavía rige fuertemente la racionalidad del funcionamiento del sector público, se ha generado una cultura de la medición del desempeño del sector público que privilegia los resultados entendidos en términos de eficiencia y eficacia. Este paradigma constituye un punto de referencia a la hora de evaluar el funcionamiento de las entidades del sector público en Colombia, independientemente del nivel de gobierno, las condiciones de las que depende la capacidad de gestión que se evalúa y el impacto generado en la calidad de vida de las comunidades. En este sen- 
tido, la capacidad institucional, tal como se concibe desde el paradigma imperante y los mecanismos de evaluación utilizados, no ha superado la segunda etapa de interpretaciones y aproximaciones que forman parte de la evolución del concepto.

\section{Resultados de la investigación}

\subsection{Medición del desempeño municipal}

Desde la década de 1990 las medidas orientadas a la generación de capacidades institucionales en Colombia se ha basado en un modelo de gestión pública influido por elementos que provienen de dos fuentes principales, como son la nueva gestión pública (NGP) con orientación a resultados y eficiencia, la cual busca la democratización de la administración pública y considera al ciudadano como el principal de los servidores públicos; y la gestión pública orientada a resultados (GPOR), que se deriva a su vez, de la NGP (DNP, 2010). En términos del Departamento Nacional de Planeación [DNP] (2010, p. 11) la GPOR es: un enfoque gerencial que busca la adaptación de las estructuras, los procedimientos, las instituciones y los resultados de una organización para que su labor se oriente a obtener resultados (productos, efectos e impactos) planeados que permitan la creación de valor público.

La preocupación por la generación de capacidades institucionales en materia de seguimiento, evaluación y producción de información vinculada con objetivos de eficiencia y efectividad se encuentra ligada con el cumplimiento de compromisos fiscales adquiridos con organismos internacionales durante la década 1980. Estos primeros esfuerzos dieron lugar a la posterior generación de una cultura y unos marcos institucionales orientados a la medición y la evaluación tanto de las decisiones de política pública como de los resultados de su implementación.

Los desarrollos posteriores a la Constitución Política de 1991 y que evidencian las nuevas tendencias en materia de gestión pública derivaron en las exigencias por la formulación de planes de desarrollo tanto a nivel nacional como territorial, cuya naturaleza se orienta a la toma de decisiones participativa y a los procesos de rendición de cuentas de los gobernantes (DNP, 2010). Articulados a estos instrumentos de gestión surgen otros complementarios que entrañan la lógica de la gestión por resultados. La formulación y manejo del presupuesto público, así como la implementación de bancos de programas y proyectos que respondan a unas metodologías y procedimientos definidos desde la norma, son algunas de las herra- mientas más importantes requeridas para garantizar la ejecución de los planes de desarrollo.

Además la Constitución Política (Art. 343 y 344) estableció la necesidad del diseño y organización de sistemas de evaluación de gestión y resultados de administración pública en relación tanto con las políticas públicas como con los proyectos de inversión. La carta magna abrió la posibilidad para que el organismo nacional de planeación y los departamentos puedan realizar la evaluación de gestión y resultados de los planes de desarrollo, tanto departamentales como municipalesy puedan participar en la preparación de los presupuestos de los municipios de acuerdo con la ley. En desarrollo de estos preceptos constitucionales, la Ley 617 de 2000 ordenó al DNP la definición y aplicación de una metodología para la medición de la gestión de los municipios y departamentos del país y la publicación de los resultados de esta medición. A su vez, la Ley 715 de 2001 estableció la evaluación en el uso de los recursos asignados a los territorios mediante el sistema general de participaciones (SGP).

En la actualidad, los mandatos establecidos mediante estas dos normas se concretan en la "Metodología para la medición y análisis del desempeño municipal" del año 2005. Esta metodología permite evaluar la gestión municipal en cuatro componentes que incluyen eficacia, eficiencia, gestión y entorno, y cumplimiento de requisitos legales.

La eficacia mide el grado de cumplimiento de las metas establecidas en los planes de desarrollo y mide los logros alcanzados por el municipio en términos de productos y resultados. La eficiencia busca determinar si el municipio está optimizando la dotación de recursos humanos, financieros y físicos que tiene disponibles para producir los servicios de salud, educación y agua potable, entre otros. El componente de requisitos legales examina el grado en que los municipios dan cumplimiento a los requisitos y condiciones establecidas por las normas legales para la gestión local en los sectores básicos (educación, salud, agua potable, etc.) en el marco de la descentralización y en la destinación de los recursos, principalmente el correspondiente al sistema general de participaciones (Ley 715 de 2001). Por último, el componente de gestión y entorno cuantifica el impacto que las variables de la gestión administrativa y inanciera y el entorno municipal pueden tener sobre los resultados de eficacia y eficiencia (DNP, 2005, p. 80). 
Cada uno de los factores de medición se encuentra, compuesto, a su vez por los siguientes aspectos:

Figura 1. Factores de medición de la Metodología para la medición y análisis del desempeño municipal

\section{Eficacia}

${ }^{\star}$ Avance plan de desarrollo

* Cumplimiento de metas en producto

\section{Eficiencia}

* Estimación de la eficiencia relativa por función de producción

* Definición de mejoras potenciales en productos e insumos

*Análisis de productividades

\begin{tabular}{l}
\hline \multicolumn{1}{|c|}{ Requisitos legales } \\
$\begin{array}{l}\text { Ley } 715 \text { de } 2001 \text { (SGP) } \\
\text { Cumplimiento de la incorporación y uso de los recursos } \\
\text { de transferencias de la Nación }\end{array}$ \\
\hline
\end{tabular}

\begin{tabular}{l} 
Gestión \\
$\begin{array}{l}\text { * Capacidad administrativa } \\
\text { * Gestión sectorial } \\
\text { * Desempeño fiscal }\end{array}$ \\
\hline
\end{tabular}

\section{Fuente: Elaborado con base en DNP 2005}

Los resultados de la medición total de los diferentes componentes se presentan en un rango entre o y 100 , donde el cero se asigna a los municipios que no cumplieron con ninguno de los requerimientos para cada uno de los componentes y 100 para aquellos municipios que cumplieron la totalidad de los mismos. En este sentido, los municipios quedan clasificados en un rango de desempeño, que se presenta como una lista que permite ubicarlos en comparación con los demás con arreglo a los resultados de las evaluaciones. Asimismo, los municipios quedan clasificados en un rango específico, correspon- diente con alguna de las siguientes categorías: "crítico", "bajo", "medio", "satisfactorio" o "sobresaliente".

De acuerdo con la medición del desempeño realizada por el DNP para elaño 2010, cerca del $60 \%$ de los municipios se ubicó en los niveles "medio", "bajo" y "crítico". Sin embargo, la categoría de "satisfactorio" fue en la que se ubicó una mayor proporción de municipios comparada con las demás. Las categorías que menos porcentaje obtuvieron fueron las de "sobresaliente" y "crítico" (Tabla 1).

\begin{tabular}{|c|c|c|}
\hline \multicolumn{2}{|c|}{$\begin{array}{c}\text { Tabla 1. Porcentaje de municipios por rango } \\
\text { de desempeño }\end{array}$} \\
$\begin{array}{c}\text { Nivel } \\
\text { de cumplimiento }\end{array}$ & Rangos & $\begin{array}{c}\text { Proporción de municipios } \\
\text { por rango(\%) }\end{array}$ \\
\hline Sobresaliente & $>80$ & 7,7 \\
\hline Satisfactorio & $>70 \mathrm{y}<80$ & 32,8 \\
\hline Medio & $>60 \mathrm{y}<70$ & 27,4 \\
\hline Bajo & $>40 \mathrm{y}<60$ & 24,9 \\
\hline Crítico & $<40$ & 7,2 \\
\hline
\end{tabular}

\section{Fuente: elaborado con base en DNP (2010b).}

Al revisar la distribución de los municipios en relación con las diferentes clasificaciones parece que es indiferente la categoría del municipio con el nivel de cumplimiento de los diferentes componentes de la medición del desempeño. Esto significa, que la capacidad institucional medida en términos de desempeño, no estaría directamente relacionada con el nivel de recursos con que cuenten los municipios. En el caso de los municipios pequeños (categorías $4^{\underline{a}}$, $5^{\underline{a}}$ y $6^{a}$ ) es mayor la cantidad que se encuentra en un nivel "satisfactorio" de cumplimiento y mucho más baja la cantidad que se ubica en un nivel "crítico". De hecho, del total de los municipios que corresponden con estas tres categorías, $68 \%$ se ubica en los tres niveles más altos y apenas $32 \%$ se encuentra en los niveles "bajo" y "crítico", como se muestra en la Tabla 2.

\begin{tabular}{|c|c|c|c|c|c|c|c|c|c|c|}
\hline \multirow{2}{*}{$\begin{array}{l}\text { Nivel de } \\
\text { cumplimiento }\end{array}$} & \multirow[t]{2}{*}{ Rangos } & \multirow{2}{*}{$\begin{array}{l}\text { Proporción de municipios por } \\
\text { rango (\%) }\end{array}$} & \multicolumn{8}{|c|}{ Categorias } \\
\hline & & & $\mathbf{E}$ & 1 & 2 & 3 & 4 & 5 & 6 & Total \\
\hline Sobresaliente & $>80$ & 7,7 & 0 & 2 & 2 & 2 & 7 & 4 & 68 & 85 \\
\hline Satisfactorio & $>70 y<80$ & 33,1 & 2 & 6 & 5 & 1 & 15 & 15 & 320 & 364 \\
\hline Medio & $>60 y<70$ & 27,2 & 2 & 2 & 3 & 5 & 3 & 22 & 262 & 299 \\
\hline Bajo & $>40 y<60$ & 24,9 & 0 & 3 & 6 & 3 & 11 & 8 & 243 & 274 \\
\hline Crítico & $<40$ & 7,2 & 0 & 1 & 0 & 0 & 6 & 3 & 69 & 79 \\
\hline & & Total & 4 & 14 & 16 & 11 & 42 & 52 & 962 & 1101 \\
\hline
\end{tabular}


Para el caso de los municipios seleccionados para este análisis todos los que corresponden al departamento de Cundinamarca presentan un índice integral "sobresaliente" y en el caso de la totalidad de los municipios de Boyacá todos se encuentran en un nivel "satisfactorio". Estos municipios ocupan una muy buena posición en comparación con el agregado de los municipios del país, porque como se refleja en la Tabla 3, la mayoría están entre los primeros 200 del país y la totalidad de los mismos se encuentran dentro de los primeros 50 de su respectivo departamento.

Sin embargo, una revisión de la realidad de los pequeños municipios permitió evidenciar debilidades importantes en materia de capacidad de gestión. Aunque el componente de "Capacidad administrativa" contenido en la metodología para la medición y análisis del desempeño municipal incluye factores como cantidad de funcionarios de planta, funcionarios de libre nombramiento y remoción y cantidad de computadores disponibles en comparación con el número de funcionarios, este componente deja de lado factores cualitativos determinantes para el adecuado ejercicio de las funciones administrativas que corresponden a los diferentes cargos.

Entre estos factores están el nivel de acumulación de funciones para cada cargo, la formación de los funcionarios en relación con las exigencias del cargo y la capacidad de acumulación de conocimiento y experiencia por parte de los servidores públicos en favor de la administración municipal. Estos factores no aparecen dentro de las medi- ciones que se han realizado históricamente a los municipios porque debido a su naturaleza no es posible introducir mecanismos claros para su cálculo, además, debido a que las mediciones ponen énfasis en los resultados, los otros factores adquieren un papel secundario en tanto los resultados sean positivos. Es necesario resaltar que en general estos diferentes aspectos, al dejar en evidencia las debilidades estructurales que afectan por igual a todos los municipios, pondrían en duda la solidez del proceso de la descentralización en Colombia, asunto que no forma parte del interés de los procesos de medición.

\section{Debilidades estructurales de los municipios pequeños en Colombia}

\subsection{Funciones de los municipios pequeños}

Desde que se dio comienzo al proceso de descentralización en la década de 1980, los municipios han sido objeto del traslado de un agregado importante de funciones, las cuales en la actualidad suman alrededor de 15 sectores $^{1}$ de intervención donde los municipios de todas las categorías tienen competencia. Todos estos sectores están relacionados con la asignación directa de bienes y servicios cuyos beneficios son de alcance local, y por ende su naturaleza es municipal. Estas funciones, de acuerdo con las normas, están sujetas a su vez a su financiación mediante los recursos propios de los municipios, y en el caso de los sectores de salud, educación y agua potable, la nación ha fijado un sistema de transferencias que introduce diferencias en materia de intervención y asignación de recursos

Tabla 3. Municipios analizados por el proyecto ubicados por índice y posición nacional y departamental en el rango de desempeño

\begin{tabular}{|c|c|c|c|c|c|c|c|c|c|}
\hline $\begin{array}{c}\text { Municipio y } \\
\text { departamento }\end{array}$ & Eficacia & Eficiencia & $\begin{array}{c}\text { Requisitos } \\
\text { legales }\end{array}$ & $\begin{array}{l}\text { Capacidad } \\
\text { activa }\end{array}$ & Fiscal & Gestión & $\begin{array}{l}\text { Índice } \\
\text { integral }\end{array}$ & $\begin{array}{l}\text { Posición } \\
\text { nacional }\end{array}$ & $\begin{array}{l}\text { Posición } \\
\text { dptal }\end{array}$ \\
\hline Cundinamarca-Arbeláez & 97,0 & 58,52 & 99,4 & 96,92 & 70,89 & 83,91 & 84,7 & 26 & 17 \\
\hline Cundinamarca-Sesquilé & 96,6 & 59,95 & 87,6 & 91,88 & 79,24 & 85,56 & 82,4 & 49 & 31 \\
\hline Cundinamarca-Suesca & 98,2 & 66,62 & 93,3 & 84,81 & 75,56 & 80,19 & 84,6 & 31 & 21 \\
\hline Cundinamarca-Tenjo & 99,0 & 56,06 & 95,9 & 91,37 & 89,81 & 90,59 & 85,4 & 20 & 13 \\
\hline Boyacá-Belén & 98,9 & 52,57 & 76,9 & 87,85 & 74,71 & 81,28 & 77,4 & 163 & 11 \\
\hline Boyacá-Cómbita & 81,0 & 62,24 & 85,5 & 93,56 & 70,90 & 82,23 & 77,8 & 153 & 9 \\
\hline Boyacá-Nobsa & 77,7 & 51,59 & 96,7 & 83,68 & 89,87 & 86,77 & 78,2 & 136 & 7 \\
\hline Boyacá-Samacá & 79,6 & 57,64 & 88,8 & 68,58 & 73,67 & 71,12 & 74,3 & 292 & 32 \\
\hline Boyacá-Tibasosa & 94,0 & 39,44 & 89,1 & 92,23 & 87,33 & 89,78 & 78,1 & 139 & 8 \\
\hline
\end{tabular}

1. Estos sectores son educación, salud, deporte y recreación, cultura, infraestructura y transporte, ambiente, agua potable y saneamiento básico, ordenamiento territorial, vivienda, prevención y atención de desastres, promoción del desarrollo, fortalecimiento institucional, turismo, juventud y desarrollo agropecuario. 
dependiendo de la capacidad de la entidad territorial del nivel local.

Sobre la participación de los recursos en relación con las obligaciones de gasto asignadas a los municipios, el DNP indica en su informe del año 2011 sobre el desempeño fiscal de los departamentos y los municipios, que excluyendo a Bogotá, cuarenta municipios y doce departamentos, es cada vez menor el peso de los recursos propios dentro de los ingresos totales de las entidades territoriales, de lo que se deduce que la dependencia en los recursos provenientes de fuentes externas como son el SGP y las regalías es cada vez más alta tanto en municipios como en departamentos. En el caso de los municipios pequeños la cantidad de recursos provenientes de fuentes externas es cercana al $80 \%$ del total de sus recursos (Gráfica 1).

Gráfica 1. Participación de las diferentes fuentes de recursos en los gastos de los municipios de las categorias 4ta, 5 ta y 6 ta

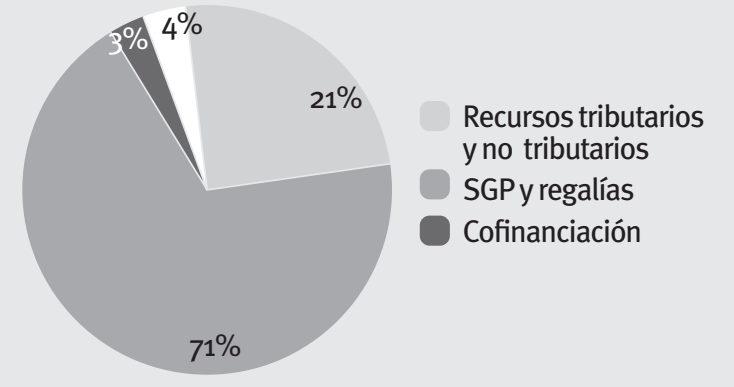

Fuente: elaborado con base en DNP (2011).
Estas limitaciones en materia fiscal se reflejan en la práctica, en unas estructuras administrativas insuficientes, porque se encontró que los municipios analizados cuentan con un promedio de 5 secretarías y unas plantas de personal reducidas (Tabla 4) donde se concentran la totalidad de las funciones asignadas, tanto desde lo misional como desde lo operativo ${ }^{2}$.

\subsection{Condiciones para el ejercicio de las funciones asignadas}

Además de los funcionarios de planta de la administración central (Tabla 4), la gran mayoría de los municipios estudiados cuenta con una planta "paralela" compuesta por servidores públicos vinculados mediante contratos de prestación de servicios, la cual tiende a igualar el número de funcionarios de planta. La presencia de esta modalidad de vinculación está justificada en el 100\% de los casos por la gran cantidad de actividades que debe asumir la entidad para garantizar el cabal desempeño de la administración municipal.

Además de la totalidad de servidores de que disponen estas administraciones, 20\% alcanza hasta un nivel de secundaria en su formación, y dentro del $80 \%$ restante, se destacan los funcionarios con nivel profesional (41\%) y nivel tecnológico (31\%). Apenas $6 \%$ ha realizado algún tipo de posgrado. Entre los títulos de formación conseguidos por los diferentes servidores consultados se destacan en nivel de importancia

\begin{tabular}{|l|c|c|c|c|}
\hline \multicolumn{1}{|c|}{$\begin{array}{c}\text { Tabla 4. Total de funcionarios por municipio } \\
\text { Departamentos - } \\
\text { Municipio }\end{array}$} & $\begin{array}{c}\text { Total } \\
\text { funcionarios } \\
\text { directivos de } \\
\text { LNR y elección }\end{array}$ & $\begin{array}{c}\text { Total cargos planta } \\
\text { nivel directivo, } \\
\text { asesor y profesional }\end{array}$ & $\begin{array}{c}\text { Funcionarios planta } \\
\text { admón central nivel } \\
\text { directivo, asesor, } \\
\text { profesional ytécnico } \\
\text { que cuentan con PC }\end{array}$ & $\begin{array}{c}\text { Funcionarios planta } \\
\text { admón central nivel } \\
\text { directivo, asesor, } \\
\text { profesional y } \\
\text { técnico }\end{array}$ \\
\hline Cundinamarca-Arbeláez & 10 & 11 & 11 & 11 \\
\hline Cundinamarca-Sesquilé & 7 & 8 & 13 & 13 \\
\hline Cundinamarca-Suesca & 6 & 14 & 20 & 20 \\
\hline Cundinamarca-Tenjo & 9 & 18 & 32 & 32 \\
\hline Boyacá-Belén & 4 & 8 & 9 & 9 \\
\hline Boyacá-Cómbita & 3 & 7 & 7 & 7 \\
\hline Boyacá-Nobsa & 5 & 10 & 16 & 16 \\
\hline Boyacá-Samacá & 6 & 26 & 14 & 28 \\
\hline Boyacá-Tibasosa & 8 & 11 & 14 & 14 \\
\hline
\end{tabular}

2. De acuerdo con lo establecido en la Ley 617 de 2000 los municipios deben cumplir con unos gastos de funcionamiento ajustados a unos porcentajes específicos en relación con sus ingresos corrientes de libre destinación. Esto conduce a que en la práctica los municipios con menos recursos deban funcionar con plantas de personal bastante reducidas. 
los de administración de empresas, contaduría pública y derecho. En menor medida se encontraron profesionales en sicología, ciencia política, arquitectura y zootécnica. En materia de posgrados se encontró un solo caso para administración pública, y otro para gerencia de instituciones de salud y derecho de familia.

Evidentemente, la fundamentación en administración pública o cualquiera de los diferentes temas que la componen no es la constante en la formación de los servidores públicos a nivel municipal. Como es de suponerse, la mayoría (68\%) de los casos los individuos consultados, incluyendo a los alcaldes y sus secretarios de despacho, coinciden en afirmar que la experiencia en el ejercicio del cargo o en cargos anteriores similares, ha sido la base principal para la adquisición de los conocimientos necesarios para el desempeño de sus funciones. Solo $24 \%$ de los que tiene alguna carrera técnica o profesional, le atribuye alguna importancia a los conocimientos adquiridos en la universidad, en especial las personas que tienen formación en derecho, por considerarse, con razón, que el conocimiento y manejo de las leyes constituye una ventaja para entender y adaptarse más fácilmente a la lógica de la administración pública.

En general, los cargos más importantes, porque son los que reúnen a los funcionarios con mayor nivel de formación en un municipio pequeño son los que corresponden al alcalde y el gabinete de gobierno. Esta situación conduce a que en la práctica se produzca una fuga de capacidad institucional cada vez que ocurre un cambio de gobierno, lo cual evidencia que la capacidad institucional en lugar de tener un comportamiento ascendente, se caracteriza por una tendencia cíclica en la medida que crece durante el período de gobierno y decrece en la transición de un gobierno a otro.

Las debilidades en la realidad administrativa de los municipios pequeños están expresadas en la existencia de falencias en la formación de los funcionarios, el carácter "promiscuo" de los miembros del gabinete de gobierno quienes deben asumir bajo su autoridad varias funciones al mismo tiempo, y finalmente en la complejidad y mutación permanente inherentes con la naturaleza de los ámbitos de actuación de la administración pública. A pesar de que esta situación demuestra una necesaria intervención, no se encontró un proyecto claro de fortalecimiento permanente orientado hacia las administracio- nes municipales. De hecho, es indudable que las actividades para el fortalecimiento institucional de los municipios no guardan ninguna frecuencia temporal o periodicidad temática que permitan concluir sobre la existencia de un propósito claro (Tabla 5).

\begin{tabular}{|c|c|}
\hline $\begin{array}{c}\text { Capacitaciones recibidas en los } \\
\text { últimos } 3 \text { años }\end{array}$ & $\begin{array}{l}\text { Relación } \\
\text { porcentual }\end{array}$ \\
\hline Protección social & 5 \\
\hline $\mathrm{MECl}$ & 16 \\
\hline Talento humano & 8 \\
\hline Contratación pública & 13 \\
\hline Manejo de seguridad & 2 \\
\hline Impuestos & 2 \\
\hline Proyectos de inversión pública & 2 \\
\hline Presupuesto y finanzas públicas & 2 \\
\hline Asesoría contable y jurídica & 2 \\
\hline Ordenamiento territorial & 1 \\
\hline Manejo de archivos & 7 \\
\hline Procesos y procedimientos & 4 \\
\hline Herramienta administrativa sistematizada & 4 \\
\hline Gestión empresarial & 1 \\
\hline Fortalecimiento institucional & 4 \\
\hline Atención al cliente & 7 \\
\hline Prevención y vacunación & 1 \\
\hline Población vulnerable & 1 \\
\hline Gobierno local & 2 \\
\hline Administración pública & 2 \\
\hline Estado y democracia & 1 \\
\hline Seguimiento en políticas públicas & 1 \\
\hline Servicios públicos & 2 \\
\hline Redes de ludotecas & 1 \\
\hline Veeduría ciudadana & 2 \\
\hline Agroturismo & 1 \\
\hline Mejoramiento genético & 1 \\
\hline
\end{tabular}

Debido a la amplitud del universo de temas de la administración pública y el Estado, es evidente la dispersión de temáticas sobre las cuales se han impartido capacitaciones a las administraciones locales estudiadas. La mayor proporción se ubica en los temas de $\mathrm{MECl}$ y contratación pública y en menor medida en bienestar social, talento humano, archivo y procesos y procedimientos. Así, la gran mayoría de estas capacitaciones se ha llevado a cabo por iniciativa de la administración municipal y sorprende que la participación del departamento en la oferta de este tipo de apoyos sea muy baja para el caso de los municipios analizados en el departamento de Boyacá. Este aspecto es importante en la medida que desde la norma el nivel intermedio es el llamado de manera directa a brindar apoyo y asistencia técnica y administrativa a los municipios. 
Tabla 6. Características de las capacitaciones recibidas por los municipios en los últimos 3 años

\begin{tabular}{|l|c|c|c|c|c|c|c|c|c|c|c|c|}
\hline \multirow{2}{*}{ Departamento } & \multicolumn{4}{|c|}{ Origen de la capacitación (\%) } & \multicolumn{3}{c|}{$\begin{array}{c}\text { Iniciativa de la } \\
\text { capacitación (\%) }\end{array}$} & \multicolumn{3}{c|}{ Utilidad de la capacitación (\%) } \\
\hline & DNP & Gobemación & Alcaldía & ESAP & Otro & Intema & Externa & Alta & Media & Baja & Ninguna \\
\hline Cundinamarca & 3 & 25 & 28 & 3 & 40 & 53 & 47 & 72 & 26 & 0 & 2 \\
\hline Boyacá & 0 & 9 & 56 & 16 & 19 & 74 & 26 & 96 & 4 & 0 & 0 \\
\hline
\end{tabular}

Otro factor a tener en cuenta es la existencia de una correspondencia directa entre la iniciativa de las capacitaciones y la utilidad que le atribuyeron los funcionarios municipales, porque en su mayoría el origen de las iniciativas se dio desde el interior del municipio y la utilidad de las capacitaciones fue en su mayoría calificada como alta. En estos términos, se confirma en la práctica el hecho que el desarrollo sostenible de la capacidad de gestión es posible solo cuando hay demanda efectiva de parte del gobierno local y la comunidad, además, que la asistencia técnica debe responder a la demanda local, ceñirse a las necesidades locales y prestarse en forma descentralizada (Banco Mundial, 1997) (Tabla 6).

A pesar de los diferentes esfuerzos por capacitar a los funcionarios locales, las necesidades de apoyo continúan siendo una preocupación constante. Los repetidos cambios que experimenta el diseño normativo en relación con la administración pública y las mutaciones que esto genera en el manejo de metodologías, procedimientos y herramientas tecnológicas para el cumplimiento de las funciones son un factor de presión permanente que se suma a las limitaciones en la formación del factor humano.

Al indagar sobre aquellos aspectos que permitirían mejorar el desempeño del cargo de los funcionarios una gran mayoría (68\%) sugirió la necesidad de las capacitaciones, y en menor medida se plantearon necesidades de dotación (17\%) y acompañamiento (15\%). Los temas más recurrentes en materia de capacitación lo constituyen las actualizaciones normativas en todos los casos, pero en particular en las materias de presupuesto y contratación. En relación con el presupuesto, la liquidación de impuestos y el manejo de la contabilidad requieren la actualización y capacitación en el manejo de software especializado. Otro de los temas que más importancia recibió fue el de atención al usuario tanto en materia de capacitación como de acompañamiento (Tabla 7).
Tabla 7. Necesidades de fortalecimiento de la capacidad de gestión municipal

\begin{tabular}{l|l|l|l} 
Temas & Software & Capacitación & Acompañamiento
\end{tabular}

\begin{tabular}{|l|l|l|l|}
\hline Impuestos & $\mathrm{x}$ & $\mathrm{x}$ & \\
\hline $\begin{array}{l}\text { Contabilidad, } \\
\text { finanzasy } \\
\text { presupuesto } \\
\text { público }\end{array}$ & $\mathrm{x}$ & $\mathrm{x}$ & $\mathrm{x}$ \\
\hline
\end{tabular}

Talento humano $\quad \mathrm{x}$

Actualización

normativa en

todos los temas

Contratación

Política pública, liderazgoy

juventud

Violencia y

sociedad, Familia,

infancia,

resolución de

conflictos

Manejo de

archivosy

trámites

$\mathrm{x}$

$x$

Atención al

usuario

Fuente: Duque, N. (2012).

Debido a las particularidades y la gran variedad de temáticas que ha introducido el diseño normativo al quehacer de las administraciones municipales, es necesario tener claridad sobre los diferentes niveles, características y frecuencias de intervención que involucra el desarrollo institucional. En estos términos, es posible encontrar temáticas que requieren de una intervención periódica en la medida que experimentan transformaciones permanentes y otras temáticas cuya atención puede ser menos frecuente. Asimismo, dependiendo de las particularidades y los niveles de necesidad de los municipios existen temáticas que requieren una o varias estrategias como la capacitación, el acompañamiento o la simple consulta.

\subsection{La importancia del municipio pequeño}

Este importante inventario de dificultades y necesidades a las que se enfrentan a diario las 
administraciones locales podría justificar los propósitos de eliminación de los municipios que no logren cierto tamaño poblacional y territorial y cierta cantidad de recursos propios. Sin embargo, aunque algunas normas, como es el caso de la Ley 617 de 2000 , han buscado la eliminación de municipios atendiendo en particular a factores de tipo fiscal, en la práctica se ha propendido por un incremento gradual, aunque lento, de estas entidades territoriales que logran subsistir, en su mayoría con el nivel mínimo requerido de recursos.

Entre los aspectos que más aportan a la justificación en el incremento de municipios pequeños en Colombia está el hecho que su existencia como entidad territorial, con autonomía, funciones y recursos propios constituye una ventaja para mejorar los niveles de cobertura y calidad en la provisión de bienes y servicios públicos. Su existencia permite, además, avanzar hacia la consolidación de un proyecto propio de comunidad que atienda las necesidades y particularidades de los habitantes del territorio, lo cual depende y alimenta, a su vez, la existencia de factores identitarios, sentido de pertenencia y lazos de cohesión social. La mayor cercanía entre las comunidades y el Estado debería conducir a un mayor conocimiento e interés de éstas por los temas públicos, garantizando la mayor intervención de la sociedad local en la gestión de sus asuntos propios en procesos que se han denominado de "cogestión".

Estos factores, sin embargo, no forman parte de la medición del desempeño a nivel territorial porque la metodología que aplica planeación nacional se orienta a la medición de procesos, gestión y resultados y su utilidad se restringe al ámbito de la organización y el funcionamiento de la administración. En este sentido, la medición del desempeño, además de ignorar factores cualitativos relevantes para la organización, deja de lado la evaluación de impacto en relación con los productos o resultados de la gestión, así como la percepción de las comunidades sobre el desempeño de la administración pública local (DNP, 2005) y los vínculos entre la comunidad y entre esta y el estado a nivel local.

En la medida que estos aspectos no sean factores de importancia a la hora de evaluar la descentralización y medir el desempeño de los municipios, no es posible identificar las falencias y tampoco definir un proyecto de fortalecimiento institucional orientado, no solo a las administra- ciones locales, sino también a las comunidades que habitan los territorios donde éstas tienen jurisdicción. Por este motivo, las comunidades desconocen, en gran medida, el papel y las responsabilidades en materia social que deben asumir a ciencia cierta las autoridades de su municipio. Además, su nivel de desconocimiento está mucho más lejos cuando se trata de los asuntos relacionados directamente con la gestión pública. Esto ha derivado en la falsa percepción acerca del municipio como un solucionador ilimitado de necesidades públicas y privadas y a la consolidación de prácticas clientelares y asistencialistas por parte de algunas autoridades en busca de mejorar sus niveles de popularidad.

En algunos casos, el desconocimiento de los límites en la actuación de sus autoridades hace que las comunidades eleven solicitudes sobre asuntos que no forman parte de las responsabilidades municipales, que no son posibles por las limitaciones de recursos o que se orientan a solucionar problemas particulares. Las autoridades tienden en algunos casos a deformar la destinación de los recursos públicos con fines clientelistas, lo cual contribuye a la deformación en la percepción de la sociedad sobre el verdadero papel de sus gobernantes y el uso de los recursos públicos.

En estos términos, la ausencia de una sociedad madura en el ámbito local que permita el desarrollo de verdaderas prácticas de cogestión y de control social que presionen la evolución de las administraciones locales se debe considerar como otro de los factores en contra de la acumulación de capacidad institucional para los municipios pequeños en Colombia.

\section{Conclusiones}

Aunque las nuevas tendencias en administración pública han buscado hacer más eficientes y eficaces los organismos del Estado, en la práctica persisten diferentes factores que evidencian debilidades en las administraciones locales, las cuales escapan a la lógica de medición establecida desde planeación nacional en razón a que las mismas no afectan el logro de los indicadores tal como se encuentran definidos desde la metodología. Incluso, se encontró que la capacidad institucional, en términos de la medición que realiza el DNP, no está directamente relacionada con el nivel de recursos a nivel municipal, porque la mayoría de los municipios pequeños del país cumplió los indicadores de manera satisfactoria. 
Este hallazgo contrasta con el hecho de que la capacidad administrativa de un municipio está directamente relacionada con la cantidad y calidad de sus recursos humanos, lo cual depende en todos los casos del nivel de recursos de las entidades territoriales.

En términos generales, las razones que subyacen los defectos de las dinámicas administrativas en el ámbito local pueden encontrarse fuertemente ligadas con factores del diseño normativo de los municipios, que al darle el mismo tratamiento a estas entidades independientemente de la existencia de características diferenciadoras en materia administrativa y fiscal, terminan afectando principalmente a las de menor tamaño y menos recursos.

En estos términos, las principales dolencias de los municipios pequeños tienen que ver con la presencia de personal cuya formación de base no está ligada, en la mayoría de los casos, con los temas de la administración pública, por lo que sus conocimientos son adquiridos por las presiones derivadas del ejercicio del cargo. A esto se suma la acumulación de funciones por unidad administrativa debido a que la escasez de recursos obliga a disponer de estructuras y plantas de personal limitadas, y la ausencia de un proyecto claro de fortalecimiento de capacidades administrativas que tome en cuenta las necesidades y las particularidades de intervención frente a cada una de ellas.

En la práctica, este tipo de entidades nunca logrará lo que algunos han denominado "la mayoría de edad" debido a que en su caso la acumulación de capacidad administrativa no es ascendente sino cíclica, lo que hace que sus debilidades y necesidades de apoyo resurjan interminablemente. Sin embargo, como más del $80 \%$ de los municipios de Colombia son pequeños y la importancia en materia social y política que estos representan para sus comunidades, es necesario que se defina, desde el diseño normativo un esquema institucional que se ajuste a la realidad y verdaderas potencialidades que ofrecen este tipo de entidades.

Este proyecto debe involucrar aspectos relacionados con el diseño en materia de funciones y recursos, caracterización de niveles de capacidad instalados y requeridos para el cumplimiento de las responsabilidades asignadas por los niveles de recursos existentes, con el fin de definir estrategias de intervención tendientes al forta- lecimiento permanente de la capacidad administrativa. Finalmente, se requiere la definición clara del papel de las comunidades en los procesos de gestión pública que permitan un mayor involucramiento de éstas en los asuntos públicos y que permitan definir líneas claras de formación para la sociedad sobre los asuntos estatales.

\section{Referencias}

Aghón, G. (1999). Descentralización fiscal tendencias y tareas pendientes $\mathrm{N} N 3$, presentado en I Comité de Hacienda y Finanzas Municipales, Unión de Ciudades. Bogotá, Colombia. Agosto de 1999. Alcaldía Mayor y la Secretaría de Hacienda del Distrito. Bogotá, Colombia.

Arbós, X. y Giner, S. (1993). La gobernabilidad. Ciudadanía y democracia en la encrucijada mundial. Madrid, España: Siglo XXI de España Editorial S.A.

Banco Mundial (1997). Colombia, estudio sobre la capacidad de los gobiernos locales: más allá de la asistencia técnica en La descentralización y el desarrollo institucional en Colombia, hoy: un análisis a la descentralización. Bogotá Colombia: Escuela Superior de Administración Pública (ESAP).

Brito, M. (2002). Buen gobierno local y calidad de la democracia. Ponencia presentada en el I Congreso Latinoamericano de Ciencia Política. Salamanca, España. 9 al 11 de mayo. Universidad de Salamanca.

Cabrero, E. (2006). Los retos institucionales de la descentralización fiscal en América Latina. Revista del CLAD Reforma y Democracia, (34), (pp. 185-216).

Chávez, 0. y Rayas, I. (2006). Fortalecer la capacidad institucional. La plataforma para un gobierno exitoso, en Portal de desarrollo. Recuperado 12/06/2012 de: http://demos. estrasol.com.mx/icma/media/Competitividad_Institucional.pdf.

Colombia, Departamento Nacional de Planeación (DNP) (2011). Desempeño fiscal de los departamentos y municipios 2010. Bogotá, Colombia. Recuperado 06/02/2012 de:http://www.dnp. gov.co/LinkClick.aspx?fileticket $=8$ Y2gKqxEvg Q\%3d\&tabid $=386$ 
Colombia, Departamento Nacional de Planeación (DNP) (2010a). Evolución de SINERGIA y evaluaciones en administración del Estado. 15 años del Sistema Nacional de evaluación de Gestión y Resultados - Sinergia: Una mirada desde las evaluaciones de política pública más relevantes. Recuperado 11/02/2012 de: http:// www.dnp.gov.co/LinkClick.aspx?fileticket $=B$ $\mathrm{KO}_{3}$ S9pPTS8\%3D\&tabid=36

Colombia, Departamento Nacional de Planeación (DNP) (2010b). Escalafón de desempeño integral municipal, vigencia 2010. Recuperado 13/05/2012 de: http://www.google.com.co/ url?sa $=$ t $\& r c t=j \& q=$ escalafon $\% 20$ de $\% 20$ desempe $\% \mathrm{C}_{3} \% \mathrm{~B} 10 \%$ 2ointegral\%2omunicipal\%20vigencia\%202010\&source $=$ web \&cd $=$ $1 \&$ ved $=0$ CB8QFjAA\& url $=$ http $\% 3 A \% 2 F \% 2 F w$ ww.dnp.gov.co\%2FLinkClick.aspx\%3Ffiletick et\%3D92qZ4iQoerw\%253D\%26tabid\%3D387 \&ei=0zBjULbmC4Ow8ASb84GwCw\&usg=AFQ jCNGzucsf50oED4gffKdNxwo_BLgASw

Colombia, Departamento Nacional de Planeación (DNP) (2005). Metodología para la medición y análisis del desempeño municipal. Recuperado 21/09/2012 De: http://www.dnp.gov. co/Portals/o/archivos/documentos/DDTS/ Gestion_Publica_Territorial/1aMetod_desem_mpal.pdf

Domínguez, J. y Jiménez, P. (2010). El acceso al agua y saneamiento: un problema de capacidad institucional local. En: Gestión y política pública - Centro de Investigación y Docencia Económicas, 19 (2), (pp. 311-350).

Fondo Monetario Internacional (2002). La función del fortalecimiento de las capacidades en la lucha contra la pobreza. Recuperado 05/04/2012 de: http://www.imf.org/external/ np/exr/ib/2002/esl/031402s.htm

Hilderbrand, M. \& Grindle, M (1997) Building sustainable capacity in the public sector. what can be done? In: Getting good government. capacity building in the public sectors of developing countries. Merilee, S. Grindle, (ed.). Boston, USA: Harvard UniversityPress.
Hintze, J. (s/f). Guía para la evaluación de la capacidad institucional. Buenos Aires, Argentina: Tecnología para la Organización Pública (TOP).

Nelissen, N. (2002). The Administrative Capacity of New Types of Governance. Public Organization Review: A Global Journal, Kluwer Academic Publishers, (2), (pp. 5-22).

Ospina, S. (2002). Construyendo capacidad institucional en América Latina: el papel de la evaluación como herramienta modernizadora. Presentado en VII Congreso Internacional del CLAD sobre la reforma del Estado y de la Administración Pública. Lisboa, Portugal. 8 al 11 de octubre, Centro Latinoamericano de Administración para el Desarrollo (CLAD).

Repetto, F. (2007). Capacidad estatal, institucionalidad social y políticas públicas o la búsqueda del “tesoro perdido", En Alonso, G. Capacidades estatales, instituciones y política social. Buenos aires, Argentina: Prometeo Libros Editorial.

Rosas, A. (2008). Una Ruta metodológica para evaluar la capacidad institucional. Revista Política y Cultura (30) (pp. 119-134). México D.F., México: Universidad Autónoma Metropolitana.

Savitch, H. (1998). Global challenge and institutional capacity: or, how we can refit local administration for the next century. Administration \& Society, 30 (3), (pp. 248-273).

Unicef (1999). Literature review: Definitions of capacity building and implications for monitoring and evaluation, Draft Dec, 1999. New York, USA: Division of Evaluation, Policy and Planning, UNICEF.

Willems, S. (2004). Institucional capacity and climate actions: summary paper. París, Francia: OECD, Environmental Directorate, International Energy Agency.

World Bank (1998). Beyond the Washington consensus: Institutions Matter. Washington DC; USA. World Bank. 